\title{
Homoclinic snaking of localized states in doubly diffusive convection
}

\author{
Cédric Beaume, ${ }^{1,2, a)}$ Alain Bergeon, ${ }^{1,2, b)}$ and Edgar Knobloch ${ }^{3, \mathrm{c})}$ \\ ${ }^{1}$ Université de Toulouse, INPT, UPS, IMFT (Institut de Mécanique des Fluides de Toulouse), \\ Allée Camille Soula, F-31400 Toulouse, France \\ ${ }^{2}$ CNRS, IMFT, F-31400 Toulouse, France \\ ${ }^{3}$ Department of Physics, University of California, Berkeley, California 94720, USA
}

(Received 27 April 2011; accepted 14 July 2011; published online 6 September 2011)

\begin{abstract}
Numerical continuation is used to investigate stationary spatially localized states in two-dimensional thermosolutal convection in a plane horizontal layer with no-slip boundary conditions at top and bottom. Convectons in the form of 1-pulse and 2-pulse states of both odd and even parity exhibit homoclinic snaking in a common Rayleigh number regime. In contrast to similar states in binary fluid convection, odd parity convectons do not pump concentration horizontally. Stable but time-dependent localized structures are present for Rayleigh numbers below the snaking region for stationary convectons. The computations are carried out for (inverse) Lewis number $\tau=1 / 15$ and Prandtl numbers $\operatorname{Pr}=1$ and $\operatorname{Pr} \gg 1$. (C) 2011 American Institute of Physics. [doi:10.1063/1.3626405]
\end{abstract}

\section{INTRODUCTION}

The term doubly diffusive convection refers to convective motions in which density variations are caused by two competing components with different rates of diffusion such as temperature and solute concentration. In oceanography, when warm salty water overlies cold and fresh water, the convective instability is responsible for the presence of salt fingers. ${ }^{1,2}$ In contrast, when cold fresh water overlies warm salty water, the instability manifests itself as an oscillatory instability that sets in once the imposed temperature difference exceeds a critical threshold. Similar phenomena are found during crystal growth and solidification ${ }^{3}$ or in geophysics, where it plays an important role in magma chamber dynamics as well as in the layer above the core-mantle boundary. ${ }^{4}$ Many instabilities arising in astrophysics, such as the Goldreich-Schubert-Fricke instability, are also doubly diffusive in nature. ${ }^{5}$ Doubly diffusive convection has been extensively studied in the past and the literature covers a wide range of configurations. These include both horizontal layers in which the imposed temperature and/or concentration gradients are parallel to the buoyancy force and vertical configurations in which the gradients are perpendicular to the buoyancy force. Natural convection in a vertically stratified layer driven by a horizontal temperature gradient also exhibits doubly diffusive behavior.

The present paper focuses on doubly diffusive convection in a horizontal layer of bottom-heavy fluid that is heated from below. This configuration is the classic configuration in which convection sets in via overstability ${ }^{6}$ and the resulting oscillations may coexist with strongly subcritical but steady convection. ${ }^{7}$ Recent work of Batiste et al. ${ }^{8}$ and Bergeon and Knobloch ${ }^{9}$ showed that steady subcritical convection favors the presence of spatially localized convective structures

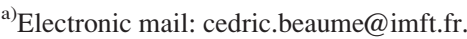

${ }^{b)}$ Electronic mail: alain.bergeon@imft.fr.

c)Electronic mail: knobloch@berkeley.edu.
}

called convectons and revealed that such structures are present in a well-defined parameter interval called the snaking or pinning region. The snaking region contains two or four branches of time-independent localized states that grow in length by nucleating additional cells on either side when the convectons are numerically followed. The new cells are nucleated at the left edge of the snaking region and reach full strength by the time the right edge is reached, where the convecton branch turns back towards smaller Rayleigh numbers and the process repeats. These back and forth oscillations of the convecton branches are referred to as homoclinic snaking. ${ }^{10}$ The resulting structures may be thought of as bound states of a pair of fronts each of which is pinned to the periodic structure in between. These fronts connect the periodic state to the spatially homogeneous conduction state and represent heteroclinic orbits between a fixed point and a periodic orbit in a spatial-dynamics interpretation of these structures. ${ }^{11}$ Both binary mixture convection in a horizontal layer heated from below ${ }^{8,12}$ and natural doubly diffusive convection driven by competing horizontal gradients ${ }^{9}$ exhibit such localized structures, and the resulting structures may be stable even when the fronts connect the state to an unstable background state, provided the instability is only convective. ${ }^{8}$

In the present work, we study standard doubly diffusive convection in two dimensions from this point of view. We are indeed able to find time-independent spatially localized structures of odd and even parity in this system and show that both one-pulse and two-pulse localized states are organized in the same type of snaking structure as in binary fluid convection or natural doubly diffusive convection. However, we find two significant differences. In contrast to binary fluid convection in which the vertical flux of the concentration necessarily vanishes along the horizontal boundaries, ${ }^{8,12}$ in the doubly diffusive system it is the concentration that is maintained at the boundaries, and it is this fact that is responsible for decreasing the effects of horizontal concentration pumping that are responsible for marked differences between odd and even parity convectons in binary fluid convection. 
In addition, we find that for the parameter values we use the snaking region lies inside the region of absolute instability of the conduction state so that all time-independent convectons are unstable. We use direct numerical simulations to identify a variety of spatially localized but time-dependent states in regions of both convective and absolute instability and speculate about their relation to the unstable convectons present at larger Rayleigh numbers. Some of these states resemble structures found in earlier simulations ${ }^{13}$ and in experiments. ${ }^{14}$

This paper is organized as follows. In Sec. II, we summarize the basic equations and their properties. In Sec. III, we present our results on one-pulse and two-pulse convectons. Section IV is devoted to time-dependent states. Brief conclusions follow in Sec. V.

\section{FORMULATION}

We consider a miscible fluid mixture, such as salt dissolved in water, in a horizontal layer $0 \leq z \leq h$ confined between a pair of no-slip horizontal boundaries, with the boundary at $z=0$ maintained at a constant temperature $T_{r}^{*}+\Delta T$ and concentration $C_{r}^{*}+\Delta C$, while the boundary at $z=h$ is maintained at $T_{r}^{*}$ and $C_{r}^{*}$, and focus on the case $\Delta T>0, \Delta C>0$. Here, $C$ is the concentration of the heavier molecular weight component. Periodic boundary conditions with period $L \gg h$ in the horizontal are imposed. The governing equations are nondimensionalized using $h$ as the unit of length and the thermal diffusion time in the vertical, $h^{2} / \kappa$, as the unit of time; $\Delta T$ and $\Delta C$ are used to nondimensionalize the temperature and concentration. The resulting dimensionless equations take the form

$$
\begin{gathered}
\operatorname{Pr}^{-1}\left(\partial_{t} \mathbf{u}+(\mathbf{u} \cdot \nabla) \mathbf{u}\right)=-\nabla p+\nabla^{2} \mathbf{u}+\left(\operatorname{Ra} T-\operatorname{Ra}_{S} C\right) \mathbf{e}_{\mathbf{z}} \\
\nabla \cdot \mathbf{u}=0 \\
\partial_{t} T+(\mathbf{u} \cdot \nabla) T=\nabla^{2} T, \\
\partial_{t} C+(\mathbf{u} \cdot \nabla) C=\tau \nabla^{2} C,
\end{gathered}
$$

where $\mathbf{u} \equiv(u, w)$ is the two-dimensional velocity field in $(x, z)$ coordinates, $p$ is the pressure, and $T$ and $C$ refer to the temperature and concentration departures from their reference values. In writing these equations, we have ignored crossdiffusion (i.e., the Soret and Dufour effects) and used the Boussinesq approximation with the fluid density $\rho$ given by

$$
\rho\left(T^{*}, C^{*}\right)=\rho_{0}+\rho_{T}\left(T^{*}-T_{r}^{*}\right)+\rho_{C}\left(C^{*}-C_{r}^{*}\right),
$$

where $T^{*}$ and $C^{*}$ are, respectively, the dimensional temperature and concentration, and $\rho_{T}<0$ and $\rho_{C}>0$ denote the thermal and solutal "expansion" coefficients at the reference temperature $T_{r}^{*}$ and concentration $C_{r}^{*}$. The Prandtl number $\operatorname{Pr}$, the (inverse) Lewis number $\tau$, and the thermal and solutal Rayleigh numbers $R a>0$ and $R a_{S}>0$ are defined by

$$
\begin{aligned}
& \operatorname{Pr}=\frac{\nu}{\kappa}, \quad \tau=\frac{D}{\kappa}, \quad R a=\frac{g\left|\rho_{T}\right| \Delta T h^{3}}{\rho_{0} \kappa \nu}, \\
& R a_{S}=\frac{g \rho_{C} \Delta C h^{3}}{\rho_{0} \kappa \nu},
\end{aligned}
$$

where $\nu$ is the kinematic viscosity, $\kappa$ is the thermal diffusivity, $D$ is the solutal diffusivity, and $g$ is the gravitational acceleration. These equations are to be solved subject to the boundary conditions

$$
\begin{gathered}
\text { at } z=0: \quad u=w=T-1=C-1=0, \\
\text { at } z=1: \quad u=w=T=C=0,
\end{gathered}
$$

together with periodic boundary conditions with period $\Gamma$ in $x ; \Gamma \equiv L / h$ is the aspect ratio of the computational domain. The above equations constitute perhaps the simplest realization of doubly diffusive convection.

The equations possess a spatially homogeneous solution

$$
u=0, \quad w=0, \quad T=1-z, \quad C=1-z,
$$

corresponding to the conduction state. In the following, we write $T=1-z+\Theta, C=1-z+\Sigma$ to describe the effects of convection and examine the properties of the equations for $u$, $w, \Theta$, and $\Sigma$. These equations are equivariant under translations in $x$ as well as under two distinct reflections

$R_{1}:(x, z) \rightarrow(-x, z), \quad(u, w, \Theta, \Sigma) \rightarrow(-u, w, \Theta, \Sigma)$,

$R_{2}:(x, z) \rightarrow(x, 1-z), \quad(u, w, \Theta, \Sigma) \rightarrow(u,-w,-\Theta,-\Sigma)$,

where $x=0$ is an arbitrarily chosen origin, hereafter the center of the domain. It follows that the equations possess the symmetry $O(2) \times Z_{2}$, where $O(2)$ is the symmetry group of a circle under rotations (translations in $x \bmod \Gamma$ ) together with the reflection $R_{1}$, while $Z_{2}$ is the symmetry group $\left\{1, R_{2}\right\}$ corresponding to symmetry under midplane reflections. The symmetry $R_{1} \in O(2)$ implies that the system is spatially reversible.

The presence of the symmetry $O(2) \times Z_{2}$ has a number of implications. It allows steady state bifurcations from the conduction state as $R a$ increases and forces these bifurcations to be pitchforks of revolution producing spatially periodic states. The symmetry also implies that any Hopf bifurcation from the conduction state leads simultaneously to branches of spatially periodic standing (SW) and traveling (TW) waves, at most one of which can be stable. ${ }^{15}$ Linear theory reveals that Hopf bifurcations are present whenever $\tau<1$ and $R a_{S}$ is sufficiently large $\left(R a_{S}>R a_{S}^{c}\right)$. The resulting SW and TW with stress-free boundaries have been studied extensively by a number of authors. ${ }^{6,16-18}$ In large domains, these waves are in turn subject to modulational instability leading to complex spatiotemporal dynamics. ${ }^{19}$

In the present work, we are interested in dynamics in large domains with no-slip boundary conditions, focusing on spatially localized structures. These are most easily studied on the real line $-\infty<x<\infty$. On this domain, the conduction state loses stability with respect to steady state perturbations at $R a=R a_{c}$. This bifurcation generates steady but subcritical periodic solutions with wavenumber $k_{c}$ obtained by minimizing the onset Rayleigh number with respect to the wavenumber $k$ of the perturbation. However, in addition to these much studied states, general theory for spatially reversible systems ${ }^{11}$ reveals the presence of a pair of (distinct) steady spatially localized states that bifurcate at $R a_{c}$ 

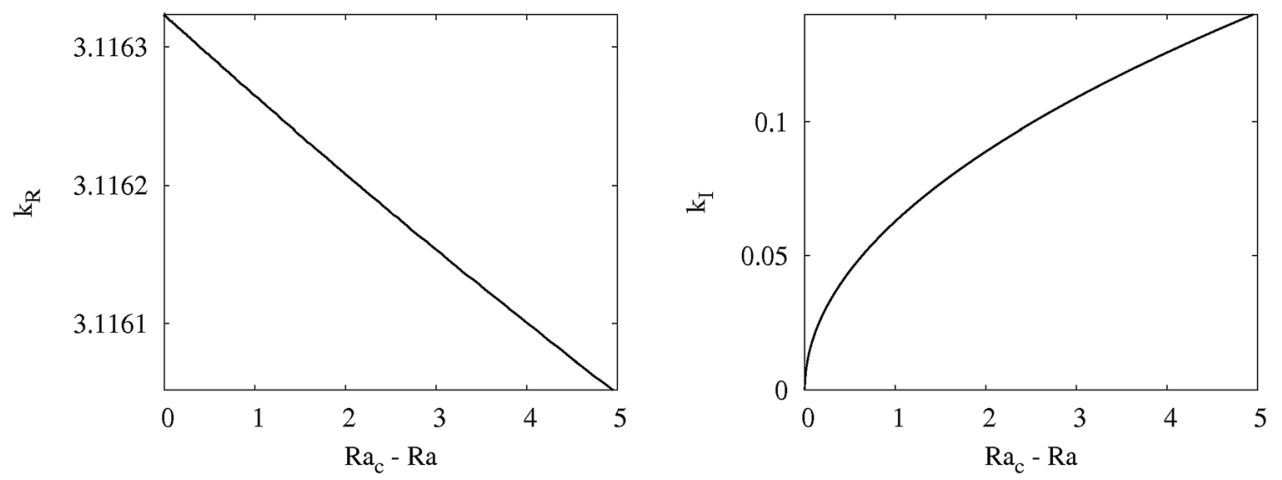

FIG. 1. The spatial eigenvalue $k \equiv$ $k_{R}+i k_{I}$ of the steady state linear stability problem in the subcritical region near $R a_{c} \approx 9207.76$. The real part of the wavenumber, $k_{R}$, varies linearly near the bifurcation point while its imaginary part varies as $\sqrt{R a_{c}-R a}$.

simultaneously with the periodic states. These states are either $R_{1}$-symmetric, viz. $(u(-x, z), w(-x, z), \Theta(-x, z)$, $\Sigma(-x, z))=(-u(x, z), w(x, z), \Theta(x, z), \Sigma(x, z))$ or $R_{1} \circ R_{2-}$ symmetric, viz. $(u(-x, 1-z), w(-x, 1-z), \Theta(-x, 1-z)$, $\Sigma(-x, 1-z))=-(u(x, z), w(x, z), \Theta(x, z), \Sigma(x, z))$. In the following, we refer to these two solution types as even and odd, respectively. Even and odd representatives of the periodic state exist also and can be obtained by suitable translation. It should be mentioned that each of these states can also be reflected in the midplane of the layer generating a new solution of the equations. This is a consequence of the fact that $R_{2}$ acts by -1 , i.e., that each solution breaks the midplane reflection symmetry.

On a domain of finite spatial period $\Gamma$, the localized states no longer bifurcate from the conduction state. Instead, these states now bifurcate from the primary branch of periodic states in a secondary pitchfork bifurcation and do so at finite amplitude; this amplitude is small when $\Gamma \gg 1$ and decreases monotonically as $\Gamma \rightarrow \infty$.

In order to compute steady solutions of the above problem as a function of the Rayleigh number $R a$, we use a numerical continuation method based on a Newton solver for the time-independent version of Eqs. (1)-(4) and boundary conditions (5)-(6). The implementation of the method follows that of Tuckerman ${ }^{20}$ and Mamun and Tuckerman ${ }^{21}$ and uses the first order time-stepping scheme proposed by Karniadakis et al. ${ }^{22}$ Details of the method in a three-dimensional context are described elsewhere. ${ }^{23}$ To discretize the equations in space, we use a spectral element method in which the domain $[0, \Gamma] \times[0,1]$ is decomposed into $N_{e}$ macro-elements of size $\left[(i-1) \Gamma / N_{e}, i \Gamma / N_{e}\right] \times[0,1]$, where $i \in\left\{1, \ldots, N_{e}\right\}$. In each element, the fields are approximated by a high order interpolant through the Gauss-LobattoLegendre points. ${ }^{24}$ Each time step requires the inversion of four Helmholtz problems and a Poisson problem for the pressure. The resulting linear systems are solved using a Schur decomposition method by separating the matrix elements acting on the nodes inside each element from those acting on nodes located along the interface between two adjacent elements. ${ }^{25-28}$ Convergence tests indicate that for our parameters calculations with $N_{e}=20$ elements, each with 17 points in the horizontal direction and 25 in the vertical direction, are well resolved. A second-order time-stepping scheme with a time step of $10^{-5}$ is used for all temporal simulations.

\section{RESULTS: TIME-INDEPENDENT STATES}

Below, we present the results for $\operatorname{Pr}=1, \tau=1 / 15$, and $R a_{S}=500$, and compare the results with the corresponding results for $\operatorname{Pr}=\infty$. This choice of parameters brackets typical parameter values for experiments on double diffusion and provides a good guide to the behavior of systems destabilized by an adverse thermal gradient for which $\tau \approx 10^{-2}$. A complementary approach, with $N \equiv R a_{S} / R a$ fixed, is commonly used in studies of natural doubly diffusive systems. ${ }^{29}$ It should be emphasized that for yet smaller Lewis numbers experiments reveal the formation of solutal boundary layers that may become unsteady at larger Rayleigh numbers. On the other hand, Lewis numbers close to unity are relevant to double diffusion in isothermal salt-sugar solution.

In Figure 1, we show the result of solving the steady state linear stability problem for the conduction solution. For this purpose, the time-independent equations are linearized about this state and solutions sought that are proportional to exp $i k x$, where $k \equiv k_{R}+i k_{I}$ is complex. The figure shows that at $R a=R a_{c} \approx 9207.76$, the wavenumber $k$ is purely real $\left(k=k_{c} \approx 3.11632\right)$ as in standard linear analysis, but that for

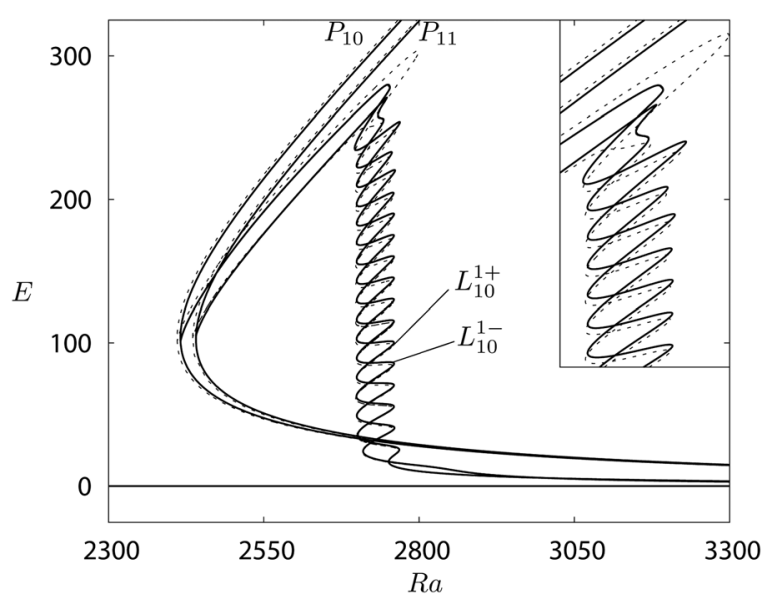

FIG. 2. Bifurcation diagram showing the kinetic energy $E$ as a function of the thermal Rayleigh number $\operatorname{Ra}$ for $\operatorname{Pr}=1$ (solid line) and $\operatorname{Pr}=\infty$ (dashed line). Only branches of interest are shown: conduction state $E=0$, periodic states $P_{10}$ and $P_{11}$, and spatially localized states $L_{10}^{1 \pm}$ of even and odd parity, respectively. Inset shows a zoom of the upper part of the snaking region. Further details are shown in Figure 3. 


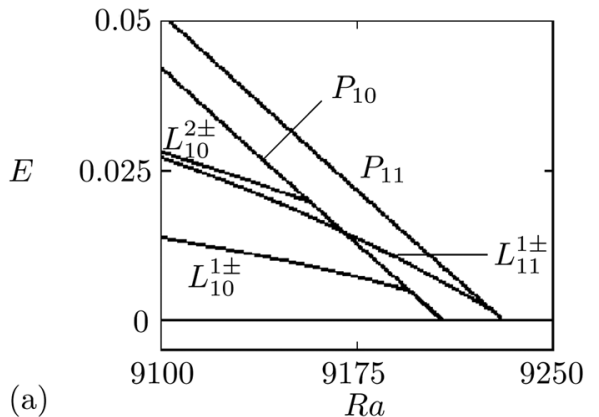

(a)

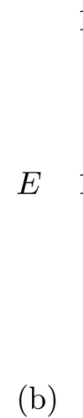

(b)

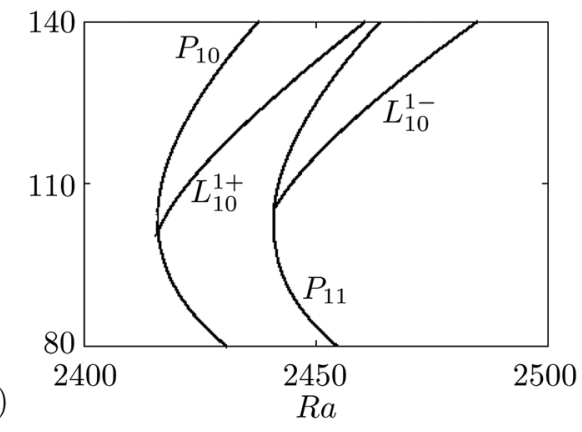

FIG. 3. (a) The vicinity of the primary bifurcation to $P_{10}$ in Figure 2 showing the secondary bifurcation from $P_{10}$ to single-pulse localized states $L_{10}^{1 \pm}$. The $L_{10}^{1 \pm}$ branches are not distinguishable on the scale of the figure. The figure also shows two families of two-pulse states $L_{10}^{2 \pm}$ and $L_{11}^{1 \pm}$ emerging from $P_{10}$ and $P_{11}$, also in secondary bifurcations. (b) The termination of $L_{10}^{1 \pm}$ on the periodic states $P_{10}$ and $P_{11}$ once the domain $\Gamma$ is filled with convection cells.
$R a<R a_{c}$, it acquires an imaginary part that increases as $\pm \sqrt{R a_{c}-R a}$, resulting in exponential growth $\left(k_{I}<0\right)$ and decay $\left(k_{I}>0\right)$ of the eigenfunction as $x \rightarrow \infty$. The presence of both growing and decaying eigenfunctions is a consequence of the spatial reversibility of the system, and both are required in order to construct time-independent exponentially localized solutions that asymptote to the conduction state as $x \rightarrow \pm \infty$. To do so, nonlinear terms must be retained. The resulting analysis reveals that, up to symmetry, there are two distinct families of such solutions, characterized by their parity: even and odd. ${ }^{11}$ The bifurcation at $R a_{c}$ corresponds to the classical exchange of stabilities in the time domain; the classical analysis shows that this exchange of stabilities point is preceded by a Hopf bifurcation at $R a \equiv$ $R a_{H} \approx 2239.67$. As already mentioned, this bifurcation generates branches of both SW and TW. Of these only the latter are computed (see Sec. IV), since the SW are expected to be always unstable.

In the following, we discuss the results of numerical continuation of both the periodic steady states and the spatially localized steady states identified in the above analysis. The calculations are carried out in a periodic domain of aspect ratio $\Gamma=10 \lambda_{c}$, where $\lambda_{c}=2 \pi / k_{c} \approx 2.0162$ is the wavelength corresponding to the critical wavenumber $k_{c}$ in an infinite horizontal layer. This spatial period, therefore, accommodates ten wavelengths of the primary unstable steady mode. Because $\Gamma$ is finite the localized states no longer bifurcate directly from the conduction state but instead bifurcate from the primary branch $P_{10}$ of periodic states in a secondary bifurcation, albeit at small amplitude. Here and in the following, we use the notation $P_{n}$ to refer to a periodic state with $n$ wavelengths within $\Gamma$ and employ the notation of Lo Jacono et al. ${ }^{12}$ to label the localized structures we find. Thus, the label $L_{n}^{m \pm}$ refers to a branch of localized solutions bifurcating from the $m$ th secondary bifurcation on the periodic state $P_{n}$, with the superscript + (resp. -) indicating solutions invariant with respect to $R_{1}$ (resp. $R_{1} \circ R_{2}$ ).

Figure 2 shows the branches $P_{10}$ and $P_{11}$ in terms of the kinetic energy $E \equiv(1 / 2) \int_{0}^{\Gamma} \int_{0}^{1}|\mathbf{u}|^{2} d x d z$, as a function of the thermal Rayleigh number $R a$. Because of the small value of $\tau$ both branches bifurcate strongly subcritically. The figure also shows a pair of intertwined branches of localized states labeled $L_{10}^{1+}$ and $L_{10}^{1-}$ with solutions that are invariant with respect to $R_{1}$ (even parity states) and the symmetry $R_{1} \circ R_{2}$ (odd parity states), respectively. As shown in Figure 3, these states bifurcate together from $P_{10}$, but terminate on different periodic states at large amplitude: $L_{10}^{1+}$ terminates on $P_{10}$, while $L_{10}^{1-}$ terminates on $P_{11}$. Thus, $L_{10}^{1 \pm}$ terminate on different branches at large amplitude although they bifurcate together at small amplitude. This behavior is a consequence of wavenumber selection across the pinning region and occurs in natural doubly diffusive convection, ${ }^{30}$ among other
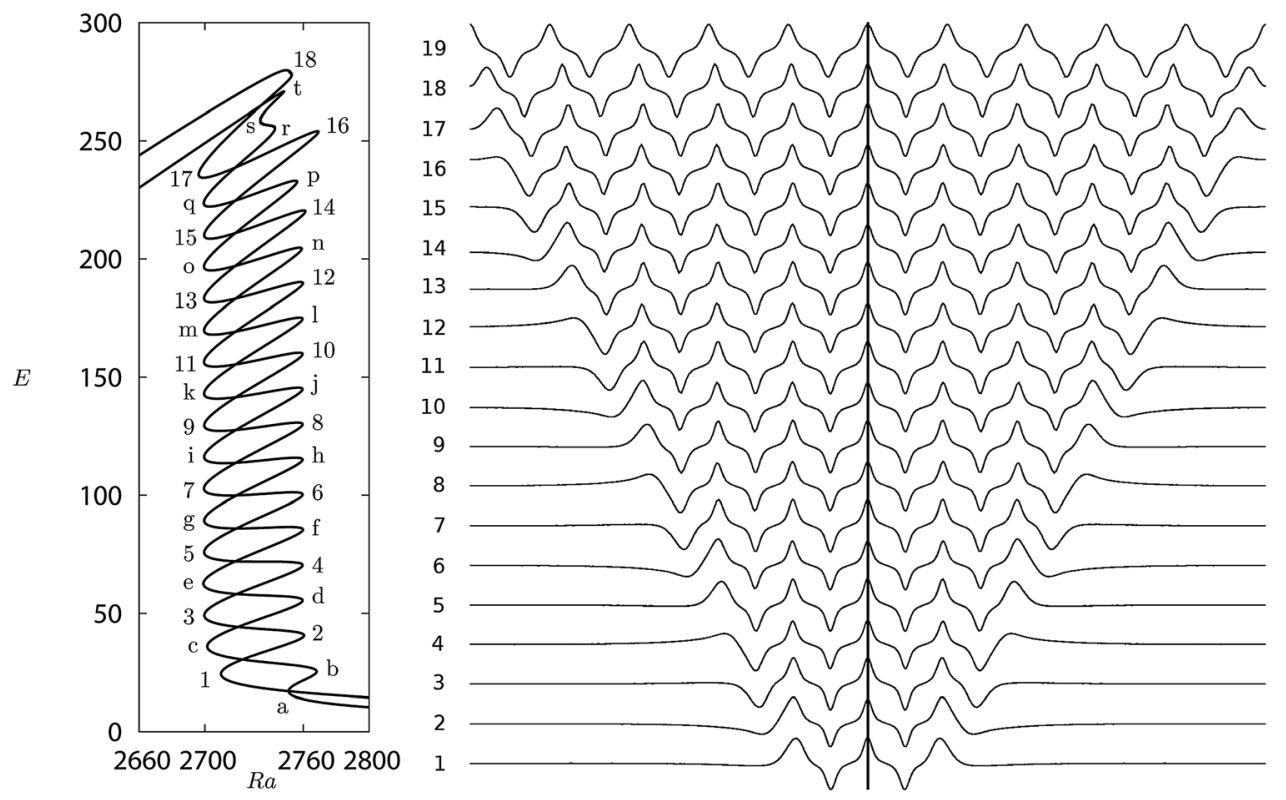

FIG. 4. (a) Detail of Figure 2 for $P r=1$ with the saddle-node bifurcations on $L_{10}^{1+}\left(L_{10}^{1-}\right)$ labeled using numbers (letters). (b) Vertically integrated concentration at the saddle-nodes of the $L_{10}^{1+}$ branch numbered as in (a). Local maxima (minima) correspond to upflow (downflow). The vertical line represents the axis of symmetry. 


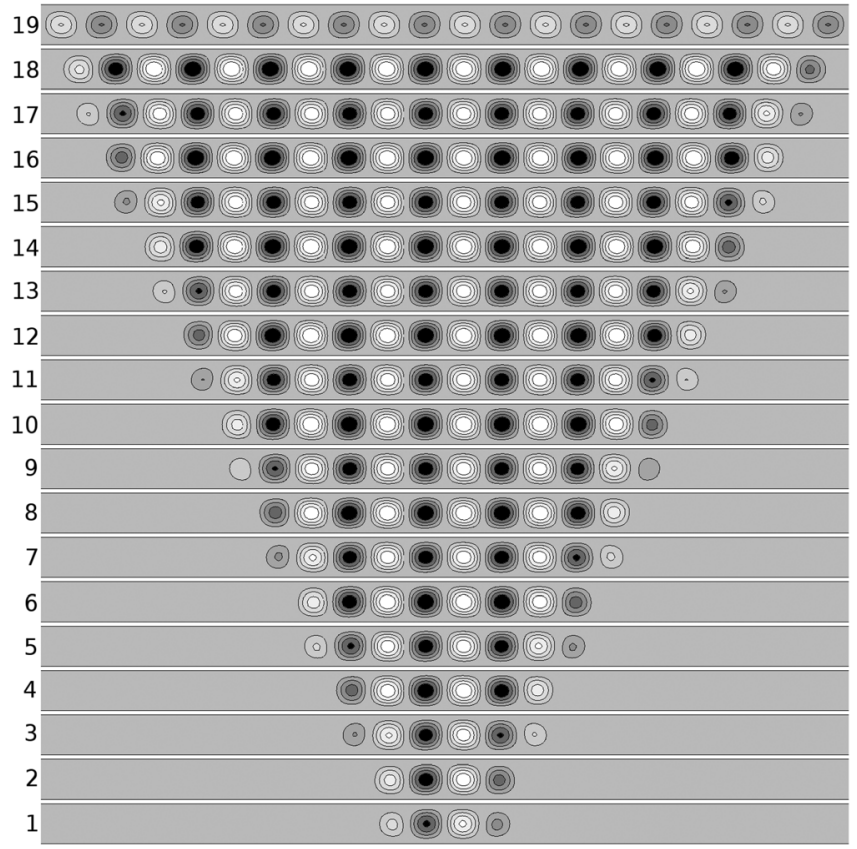

FIG. 5. Series of snapshots representing isovalues of the streamfunction at the saddle-nodes of the $L_{10}^{1+}$ branch numbered as in Figure 4(a). The structure grows by symmetrically nucleating new cells, one on each side, at $R a \approx$ $R a_{\text {left }}$, until the domain is filled with 20 rolls. Snapshot 19 is taken near the termination point of the branch (Figure $3(\mathrm{~b})$ ) on $P_{10}$, where the kinetic energy is lower.

systems. The details of the mechanism whereby the termination point of the branches of localized states switches is elucidated elsewhere. ${ }^{30}$ However, in all cases, the termination points coincide with the location of Eckhaus instability on the $P_{n}$ branches. $^{30}$

Figure 2 shows that the branches $L_{10}^{1 \pm}$ of localized states oscillate back and forth across the interval $R a_{\text {left }} \approx$ $2700<R a<R a_{\text {right }} \approx 2760$. In the following, we refer to this interval as the snaking or pinning region as in other problems, where spatially localized states are created by the same mechanism. In particular, we conjecture that $R a=R a_{\text {left }}$ corresponds to a tangency between the unstable manifold of the conduction state in space and the corresponding center-stable manifold of the periodic states, viewed as a periodic orbit in an infinite-dimensional phase space. $^{31,32}$ Likewise, $R a=R a_{\text {right }}$ corresponds to the location of a tangency between the center-unstable manifold of the periodic states and the stable manifold of the conduction state. General considerations ${ }^{11,33}$ suggest that this region also contains single-pulse states with no symmetry, found on secondary branches linking $L_{10}^{1 \pm}$ and constituting the "snakes-and-ladders" structure of the single-pulse states within this region. The $O(2)$ symmetry implies that these asymmetric states drift in the $x$-direction and so correspond to traveling pulses instead of steady states; we have not computed such states.

Figures 4(b) and 5 take a closer look at the even parity states $L_{10}^{1+}$. The figures show, respectively, the vertically integrated concentration $\int_{0}^{1} \Sigma(x, z) d z$, and the streamfunction (a pseudoscalar) as functions of $x$ at the locations indicated in Figure 4(a). The figures reveal a rising plume at the center $x=0$ with descending plumes on either side, embedded in a background conduction state. Each solution is reflectionsymmetric in $x=0$ and the width of the localized state increases in a well-defined manner as one follows $L_{10}^{1+}$ from small amplitude to large amplitude. In particular, at the leftmost saddle-nodes, labeled $1,3, \ldots$, the cells at either end of the localized structure are weak, and these strengthen as one traverses $L_{10}^{1+}$ to the next saddle-node at the right, $2,4, \ldots$, etc., where they reach maximum amplitude. Thus, the localized structure grows along $L_{10}^{1+}$ by repeatedly nucleating new cells symmetrically at both ends until the domain is almost filled. At this point, the snaking terminates and $L_{10}^{1+}$ exits the snaking region in the direction of decreasing $R a$ and terminates on $P_{10}$ (Figure 3(b)). Snapshot 19 shows that the branch terminates on an even $P_{10}$ state. Note that once created each cell remains in place. Figures $6(\mathrm{~b})$ and 7 show the
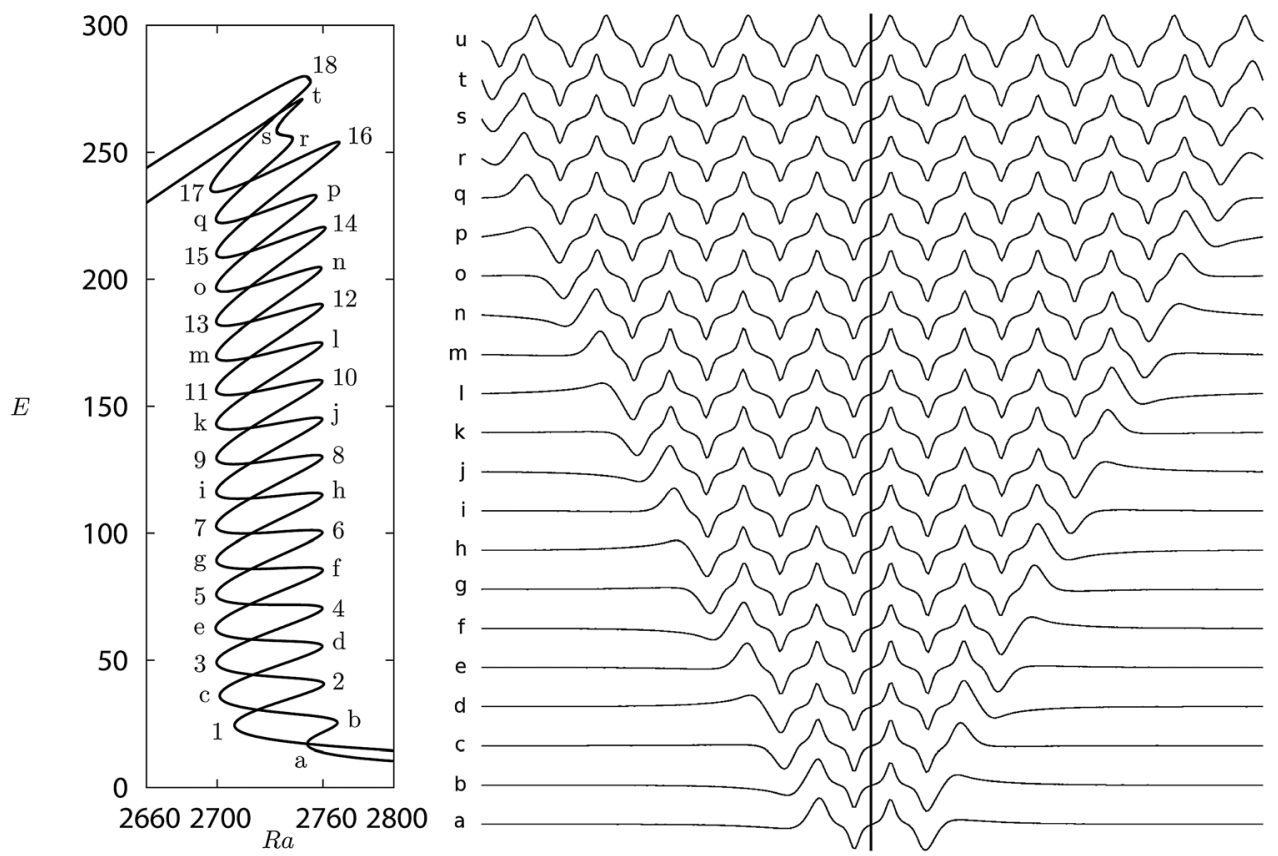

FIG. 6. (a) Detail of Figure 2 for $\operatorname{Pr}=1$ with the saddle-node bifurcations on $L_{10}^{1+}\left(L_{10}^{1-}\right)$ labeled using numbers (letters). (b) Vertically integrated concentration at the saddle-nodes of the $L_{10}^{1-}$ branch labeled as in (a). Local maxima (minima) correspond to upflow (downflow). The vertical line represents the axis of symmetry. The convectons are antisymmetric with respect to this line. 


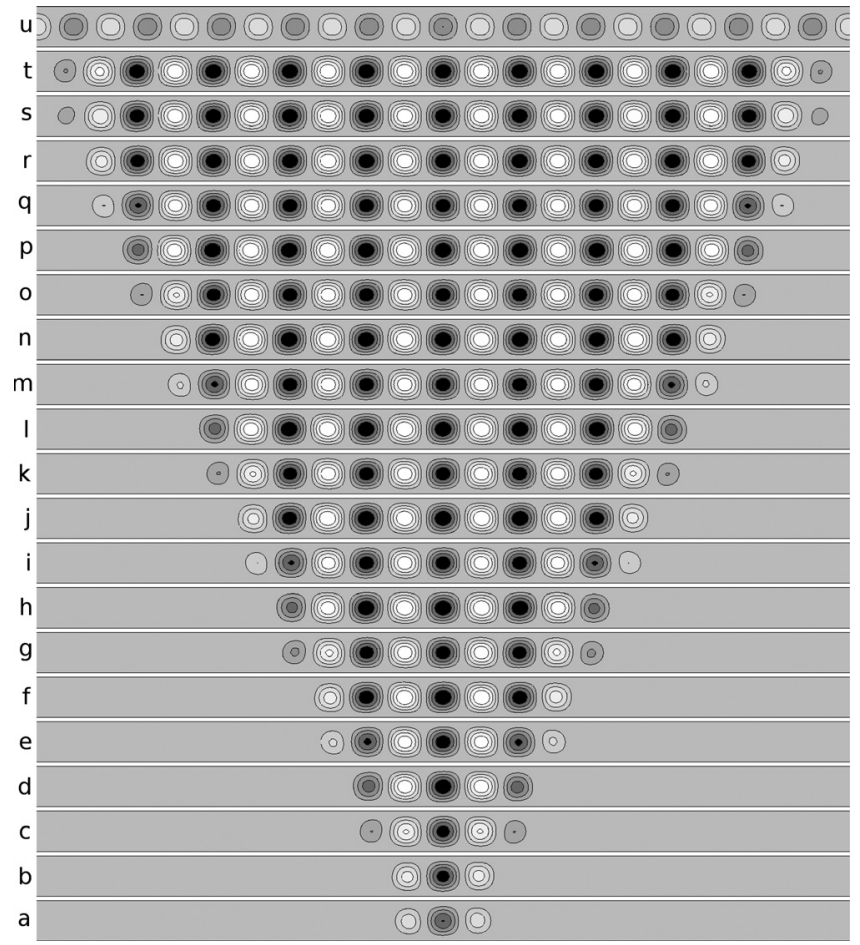

FIG. 7. Series of snapshots representing isovalues of the streamfunction at the saddle-nodes of the $L_{10}^{1-}$ branch labeled as in Figure 6(a). This time the cells nucleated on each side of the structure at $R a \approx R a_{\text {left }}$ rotate in the same direction, either clockwise (e.g., panels $a, e$ ) or counterclockwise (e.g., panels $c, g$ ) until the domain is filled with 22 rolls. Snapshot $u$ is taken near the termination point of the branch (Figure $3(\mathrm{~b})$ ) on $P_{11}$, where the kinetic energy is lower.

corresponding results for $L_{10}^{1-}$. This time each solution has point symmetry $R_{1} \circ R_{2}$, with a rising plume to the right of $x=0$ and a descending plume to the left. Once again the outer cells are weak at the left saddle-nodes labeled $a, c, \ldots$ and strengthen as one follows $L_{10}^{1-}$ to the next saddle-node at $R a_{r i g h t}$, labeled $b, d, \ldots$. Thus, the odd parity localized states again grow by nucleating cells at either end, but this time, the new cells both rotate in the same direction, for example clockwise at location $b$ (a three-cell state) and counter-clockwise at location $d$ (a five-cell state). This time, the domain fills with 11 pairs of cells since the 21 cell state labeled $t$ needs an additional cell in order to terminate on a branch of periodic state, here $P_{11}$. As shown in states $u$, termination occurs on an odd parity $P_{11}$ state. Note that in contrast to other problems of this kind, the variation in cell size or wavelength across the snaking region is almost invisible, although for both $L_{10}^{1+}$ and $L_{10}^{1-}$, the cells are slightly larger at $R a=R a_{\text {right }}$ than at $R a=R a_{\text {left }}$. This type of behavior is observed in both natural doubly diffusive convection ${ }^{29}$ and in binary fluid convection in a horizontal layer. ${ }^{8,12}$

The above results resemble corresponding results obtained for binary fluid convection in a horizontal layer. ${ }^{8,12}$ This system, while closely related to that studied here, ${ }^{34}$ differs from it in a subtle but important way. In binary fluid convection, the stabilizing concentration distribution is the result of a separation between the heavier and lighter components of the mixture in an applied temperature gradient. This effect, called the Soret effect, is important in liquids and leads to a larger concentration of the heavier component near the cold boundary (positive Soret effect) or the hot boundary (negative Soret effect). In the latter case, much studied in laboratory experiments, ${ }^{35,36}$ the destabilizing temperature gradient generates a competing stabilizing concentration gradient, much as in the present problem, where the stabilizing concentration gradient $\left(R a_{S}>0\right)$ is imposed externally, through the concentration boundary conditions. However, in binary fluids, the corresponding boundary condition is that the mass flux vanishes (impermeable boundaries), and this boundary condition imposes a condition on the concentration gradient at the boundary instead of the concentration itself. As a result a rising plume carrying solute from the lower boundary is forced to carry it horizontally when deflected by the upper boundary and conversely at the lower boundary. In contrast, in the doubly diffusive problem studied here, the rising plume collides with the boundary, decreasing the concentration length scale to such a degree that its solute content can be transported diffusively into the constant concentration reservoir used to impose the boundary condition on the concentration at the upper boundary. Thus, near the boundaries, the dominant solute transport is vertical and diffusive, while in binary fluid convection it is horizontal and advective., ${ }^{8,12}$ This fact is responsible for the absence in the present problem of horizontal pumping of concentration by odd parity convectons and explains in turn why odd and even parity convectons are found in the same snaking region. In contrast, in binary fluid convection, odd convectons at $R a_{\text {right }}$ are bounded by fully developed cells rotating in the same sense and pumping concentration from left to right (clockwise rolls) or right to left (counterclockwise rolls). This pumping is in turn responsible for shifting the right boundary of the snaking region for odd parity states towards smaller $R a$. No such shift occurs at $R a_{\text {left }}$, where the bounding cells are weak and no effective pumping takes place. As a result in binary fluid convection, the snaking region for odd parity convectons is narrower than the corresponding region for even convectons. ${ }^{8,12}$ In this sense the behavior of the doubly diffusive problem is closer to that observed in classical snaking scenarios such as that observed in the Swift-Hohenberg equation. ${ }^{11,37}$

To confirm this picture, we have computed the horizontal and vertical gradients of the concentration fluctuation $\Sigma$. The result, shown in Figure 8 for state $e$ in Figures 6 and 7, confirms that the horizontal gradients at the location of the bounding fronts are very weak compared to the vertical gradients and that any solute pumped horizontally from the convecton leaks out almost immediately via the top and bottom

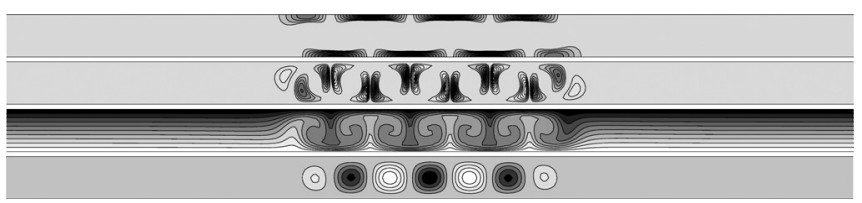

FIG. 8. The solution $e$ on the $L_{10}^{1-}$ branch. From bottom to top: isovalues of the streamfunction, the concentration $C$, and the horizontal and vertical gradients of the concentration perturbation $\Sigma$. The vertical gradients at the location of the fronts greatly exceed the horizontal gradients and any concentration pumped out of the structure immediately leaks into the constant concentration reservoirs above and below. 


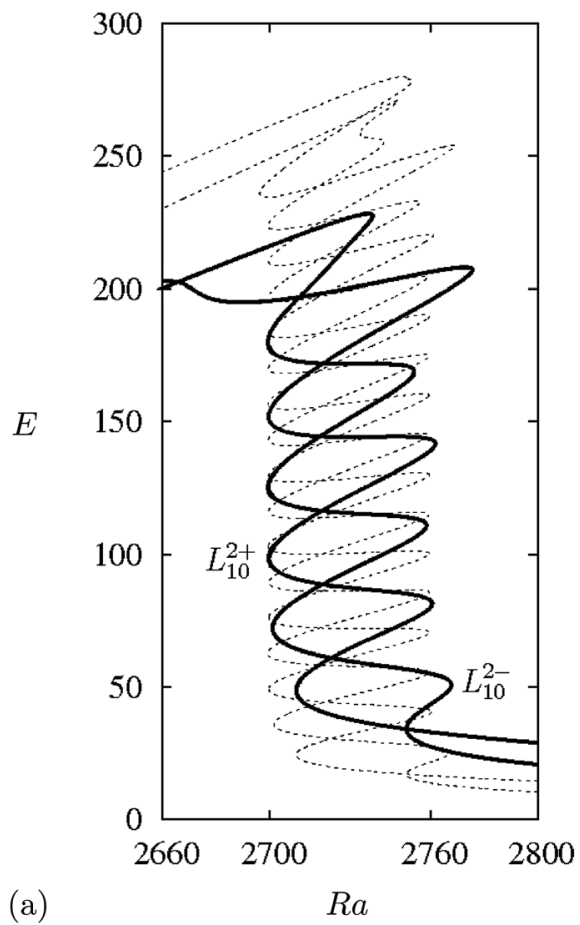

(a)

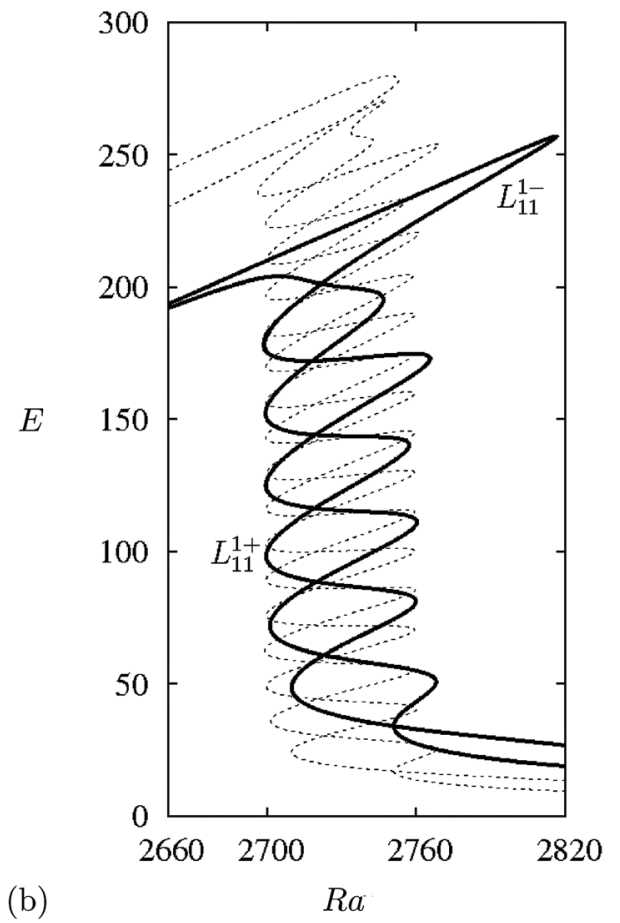

FIG. 9. (a) Snaking of the two-pulse states $L_{10}^{2 \pm}$ (bold) compared with the single-pulse states $L_{10}^{1 \pm}$ (dashed). Both sets of branches bifurcate from $P_{10}$. (b) Snaking of the two-pulse states $L_{11}^{1 \pm}$ (bold) emerging from $P_{11}$, also compared with the single-pulse states $L_{10}^{1 \pm}$ (dashed). boundaries. As a result, no large scale solute gradient is present outside the structure. Instead, the concentration is transported preferentially in the vertical direction, through the boundaries, and this is so both within the convecton and at the fronts. Thus, most of the solute brought up from the lower boundary is deposited into the lower concentration reservoir above the upper boundary and vice versa.

As in other problems of this type, the snaking region also contains numerous bound states of convectons. There are two classes of such states: states in which the convectons are equidistant when periodically replicated in the horizontal direction and states in which the convectons are clumped in groups. The former fall on solution branches that also bifurcate from periodic states and snake within the same snaking region. The latter fall on isolas as discussed further in Ref. 38 and so are harder to locate numerically. In the present work, we compute two-pulse states of the former type only.

Figure 3(a) shows two branches of two-pulse states at small amplitude. Of these, the states labeled $L_{10}^{2 \pm}$ consist of pairs of identical convectons a distance $\Gamma / 2$ apart. The convectons in $L_{10}^{2+}$ have even parity, while the convectons comprising $L_{10}^{2-}$ have odd parity, and are arranged in such a way that the resulting bound state is also even and odd. In other words, these states are nothing but a single convecton on the half domain, and it is, therefore, not surprising that $L_{10}^{2 \pm}$ branch together from $P_{10}$ just like $L_{10}^{1 \pm}$, with the $L_{10}^{2 \pm}$ branches bifurcating at a larger amplitude than the singlepulse states $L_{10}^{1 \pm}$. Figure 9(a) shows that both branches snake within the same snaking region as $L_{10}^{1 \pm}$ and that the snaking terminates after half as many folds as that for $L_{10}^{1 \pm}$. This is because the domain fills up twice as fast as for a single convecton. However, this time, the two $L_{10}^{2 \pm}$ branches terminate together on $P_{10}$ in contrast to $L_{10}^{1 \pm}$. Figure 10, top panels, shows snapshots of the corresponding solution profiles at successive saddle-nodes within the snaking region; these snapshots should be compared with the corresponding snapshots for the single-pulse states in Figures 5 and 7 . Note that the width of the snaking region for $L_{10}^{2-}$ is the same as that for $L_{10}^{2+}$, once again owing to the absence of horizontal pumping. Moreover, this region is identical to that for $L_{10}^{1 \pm}$ indicating a common origin for both sets of states. ${ }^{33}$

The snaking of the second set of spatially localized states, $L_{11}^{1 \pm}$ is shown in Figure 9(b). These branches are the product of the first secondary bifurcation on $P_{11}$ (Figure $3(\mathrm{a})$ ) and again snake within the same snaking region. Figure 10 , bottom panels, shows snapshots of the corresponding solution profiles at successive saddle-nodes within the snaking region. The solution $L_{11}^{1+}$ consists of a pair of equidistant even parity convectons, but this time with opposite sign, resulting in overall structure that is antisymmetric with respect to $x=0$. The presence of this configuration is a consequence of the midplane reflection symmetry of the system. Likewise, $L_{11}^{1-}$ consists of a pair of equidistant odd parity convectons of opposite sign, arranged back to back so that the resulting configuration is reflection-symmetric with respect to $x=0$. Like the $L_{10}^{2 \pm}$ states, the $L_{11}^{1 \pm}$ also terminate together, this time on $P_{11}$.

To explore the effect of the Prandtl number, we have recomputed the above results for $\operatorname{Pr}=\infty$. Figure 2 shows that the Prandtl number has only a very slight effect on the branches of localized states. In particular, the width of the snaking region is essentially independent of $\mathrm{Pr}$. This is in accord with the expectation that the width of this region is controlled primarily by the subcriticality of the time-independent periodic states and, hence, by the parameters $\tau$ and $R a_{S}$. This is so in binary fluid convection, ${ }^{39}$ where the width is a strong function of the separation ratio $S$, i.e., of the Soret coefficient of the mixture. 

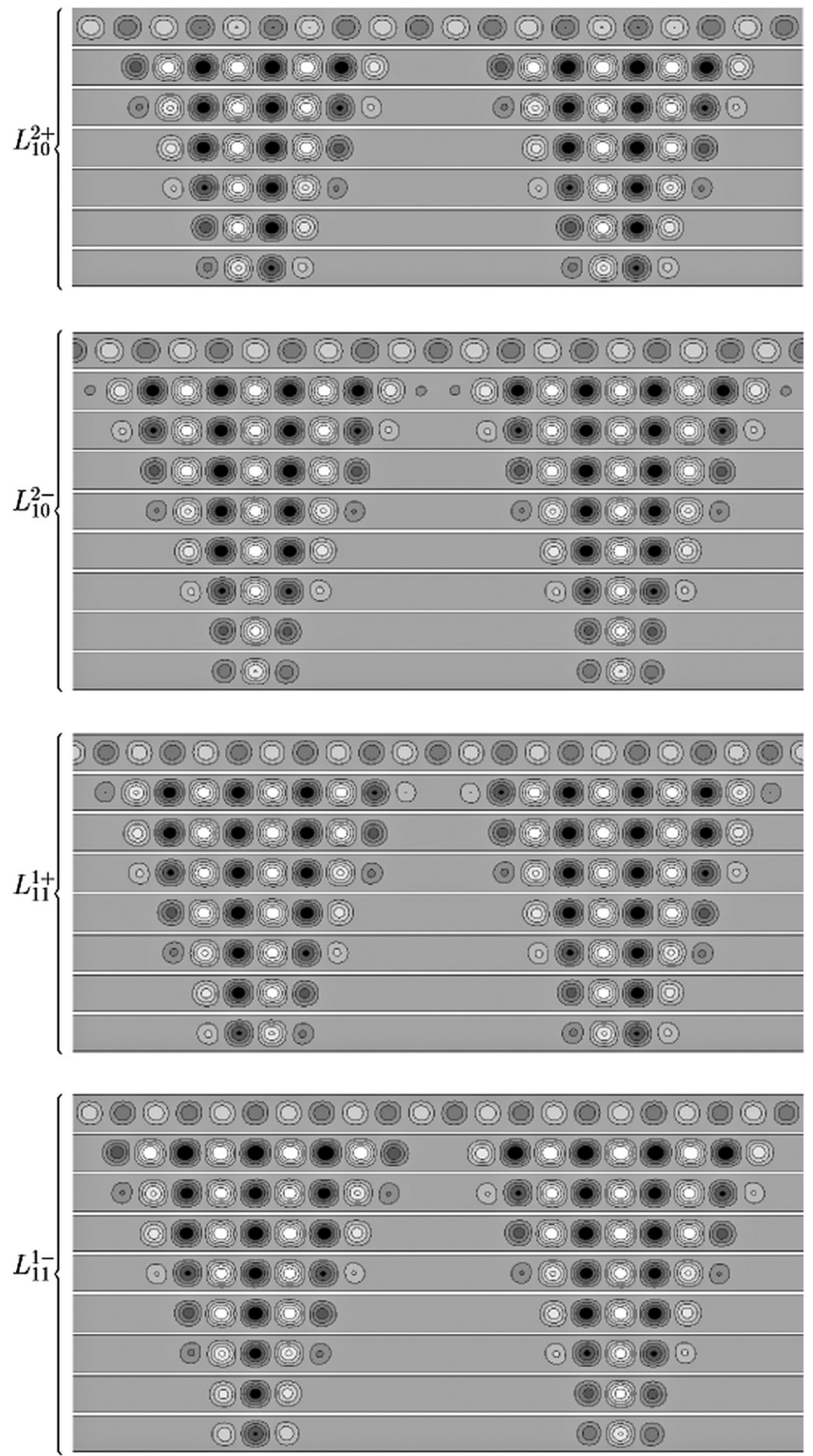

FIG. 10. Series of snapshots representing isovalues of the streamfunction at the saddle-nodes of (from top to bottom) the $L_{10}^{2+}, L_{10}^{2-}, L_{11}^{1+}$, and $L_{11}^{1-}$ branches. The last snapshot on each branch is taken near its termination point. The states $L_{10}^{2+}$ and $L_{11}^{1-}$ are even with respect to $x=0$, while $L_{10}^{2-}, L_{11}^{1+}$ are odd.

\section{RESULTS: TIME-DEPENDENT STATES}

In this section, we examine the stability of the convectons computed in Sec. III and study the dynamics of Eqs. (1)-(6), all for $\operatorname{Pr}=1, \tau=1 / 15$, and $R a_{S}=500$.

As already mentioned, the primary steady state bifurcation at $R a_{c} \approx 9207.76$ is preceded by a number of Hopf bifurcations of which the first takes place at $R a_{H} \approx 2239.67$. Figure 11 shows the resulting TW branch computed using numerical continuation. The figure shows that the TW bifurcate subcritically but quickly turn around towards larger $R a$ and thereafter increase monotonically in amplitude and terminate in a parity-breaking bifurcation on the $P_{10}$ branch at $R a=R a_{P B} \approx 2665$. Near this point, the phase speed of the TW decreases as $\sqrt{R a_{P B}-R a}$ as predicted by theory. ${ }^{15}$ Theory also predicts that when the TW bifurcate subcriti- cally, the small amplitude SW are necessarily unstable with respect to TW perturbations. Numerical simulations confirm this prediction: small amplitude spatially homogeneous SW can be found for $R a>R a_{H}$ by imposing Neumann boundary conditions on small domains but are found to decay (on a long time scale) into left- or right-traveling waves once the boundary conditions are changed into periodic boundary conditions. The direction of propagation of the resulting TW is of course arbitrary, and in the simulations is determined by numerical perturbations.

Figure 12 shows the time evolution at $R a=2300$ in a periodic domain with $\Gamma=10 \lambda_{c}$, starting from a symmetric but spatially localized perturbation as initial condition, after transients have died out. Since this $R a$ value falls below the $P_{10}$ saddle-node evolution towards steady convection is precluded. Instead, the solution evolves into a localized standing wave which subsequently breaks up into a pair of identical counter-propagating traveling waves, as expected from theory. ${ }^{15}$ Owing to the spatial periodicity of the domain, these waves necessarily collide at $x= \pm \Gamma / 2$, restoring the localized standing wave. The breakup then repeats restoring the localized SW at $x=0$. In Figure 12, this process appears to be periodic and represents a periodic attractor of Eqs. (1)-(6). Similar states have been observed in other systems as well. ${ }^{40}$

The presence of the oscillatory instability destabilizes the localized structures computed in Sec. III However, their presence appears to be responsible for a particular type of complex oscillation observed in the numerical simulations. Figure 13 shows a portion $(800 \leq t \leq 830)$ of a long time series computed at $R a=2690$ with periodic boundary conditions, starting from an odd parity one-pulse steady solution at $R a=2699.5292$ (labeled $e$ in Figures 6 and 7). The solution takes the form of counterpropagating waves colliding near $x=0$. This time the left- and right-traveling waves are $180^{\circ}$ out of phase, leading to slow zig-zag-like motion of the

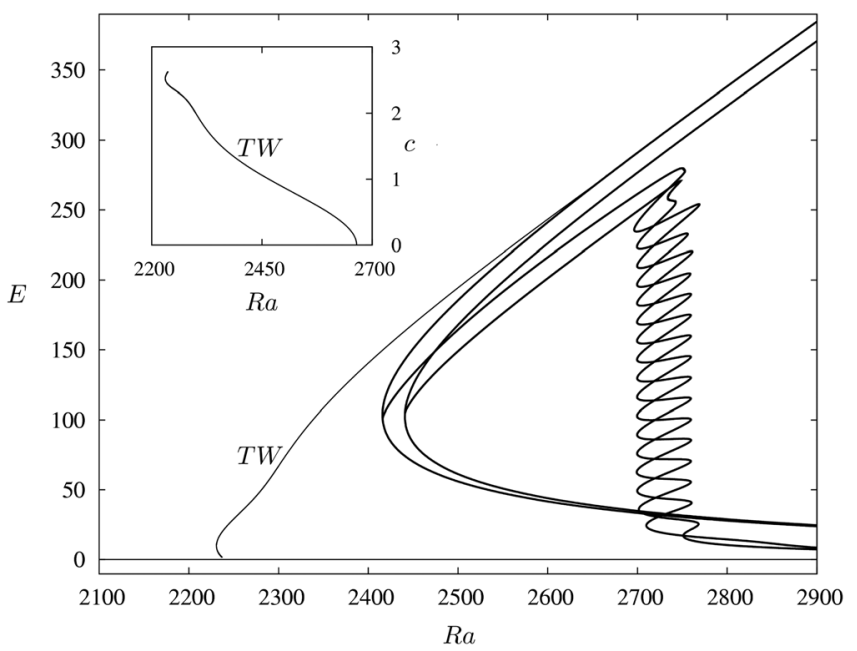

FIG. 11. The TW branch in relation to the steady states computed in Figure 2. The TW are stable between the fold at $R a \approx 2230$ and the termination point at $R a_{P B} \approx 2665$. The inset shows the phase speed $c$ along the TW branch for right traveling waves $(c>0)$; at the Hopf bifurcation at $R a_{H}=2239.67, c=\omega_{c} / k_{c} \approx 2.75$, while $c=0$ when the TW connects to the periodic branch $P_{10}$. 


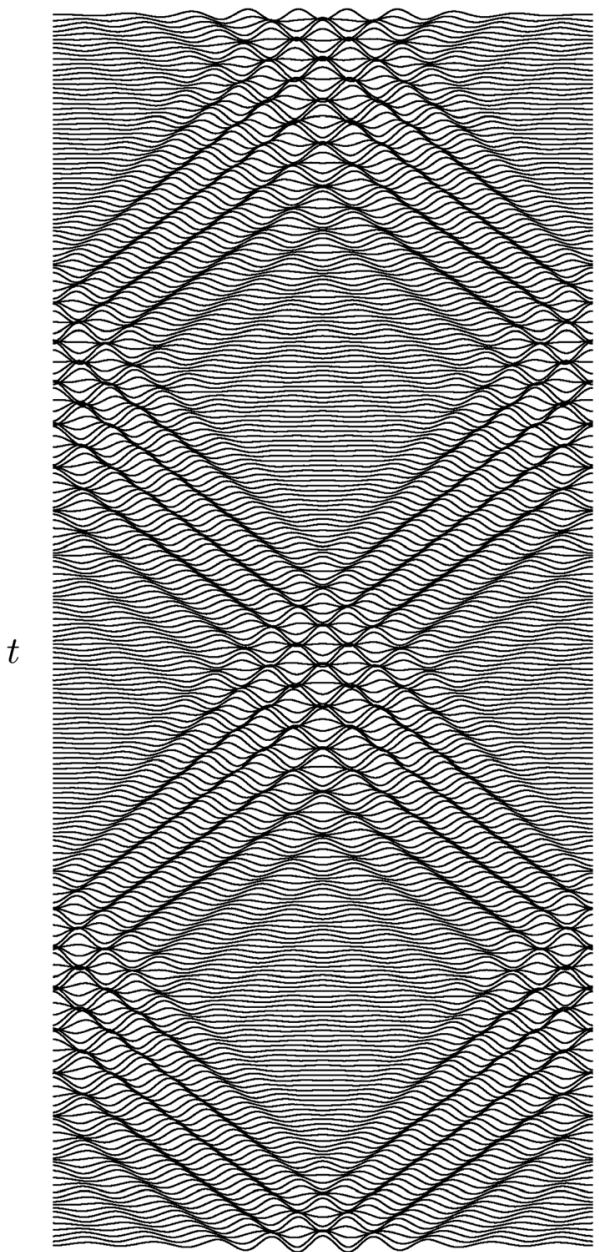

$x$

FIG. 12. Space-time plot of the midplane vertical velocity $w(x, z=1 / 2, t)$ at $R a=2300$ in the time interval $55 \leq t \leq 80$ with time increasing upward, starting from a symmetric spatially localized perturbation as initial condition. The perturbation decays into a pair of small amplitude counter-propagating traveling waves which slowly grow as they propagate. Owing to the periodicity of the domain these waves subsequently collide and reform the localized standing wave. The simulation suggests that this breakup/makeup process occurs periodically and represents a stable solution of Eqs. (1)-(6).

standing wave defect created by their collision. The resulting oscillation is nonperiodic but appears to be stable. Fourier analysis reveals the presence of frequencies associated with TW translation $(\omega \approx 0.49)$ and the switching between left and right TW $(\omega \approx 0.245)$. The same behavior is found if the computation is initialized with an even parity one-pulse state.

When $R a$ is decreased more complex dynamical behavior is found. Figure 14 shows a space-time plot of a solution obtained when $R a=2550$. The figure reveals a solution that is apparently chaotic. The left panel shows a chaotically oscillating (pulsating) source of left and right TW at $x=0$ that develops from a small amplitude spatially symmetric initial condition, together with a symmetric sink at $x=\Gamma / 2$. Because of its symmetry, the latter differs from the sink in Figure 13. The middle panel shows details of the evolution at a later time and reveals that the time-dependent source has transformed into a pulsating but still symmetric sink and vice versa for the sink. However, neither source nor sink are

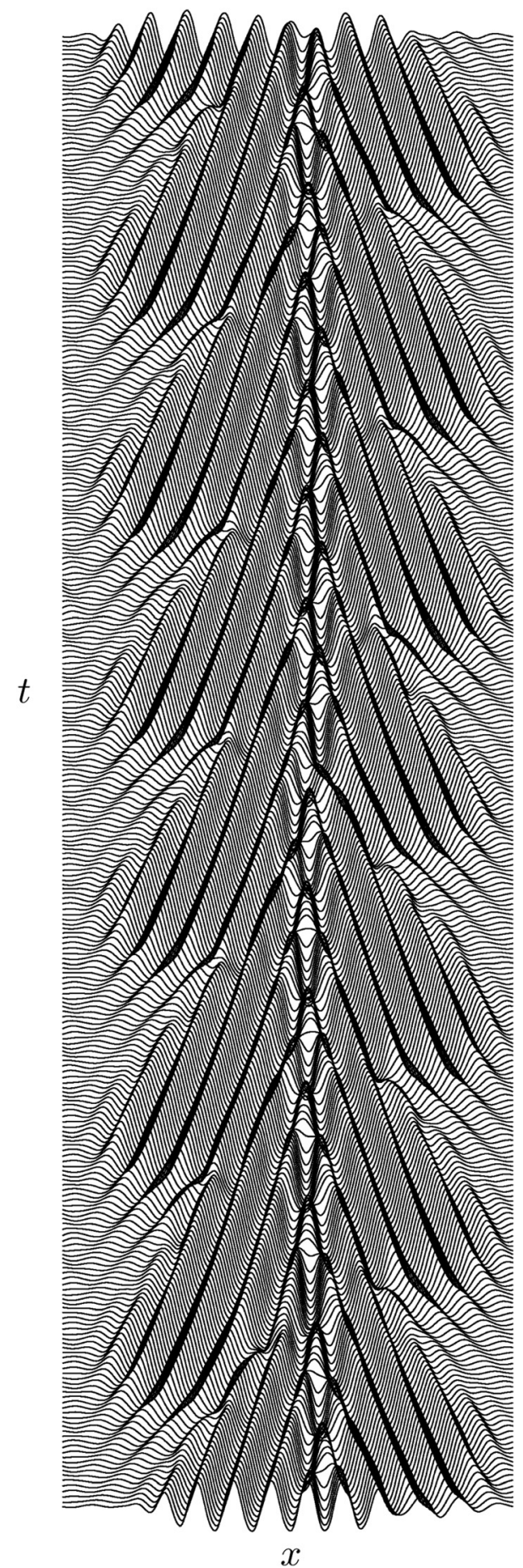

FIG. 13. Space-time plot of the midplane vertical velocity $w(x, z=1 / 2, t)$ at $R a=2690$ in the time interval $800 \leq t \leq 830$ with time increasing upward, starting from a time-independent odd parity localized state at $R a=2699.5292$, labeled $e$ in Figures 6 and 7 .

stable structures at this Rayleigh number, and on a longer timescale, the reflection symmetry of the solution is lost, and the solution evolves into a localized wave packet consisting of right-traveling waves that drifts slowly to the right (right panel). The space-time plot thus suggests the presence of a chaotic oscillation between a symmetric source and a symmetric sink that is stable if reflection symmetry is imposed on the solution (Figure 15) but unstable with respect to infinitesimal (i.e., numerically generated) symmetry-breaking perturbations when symmetry is not imposed. 


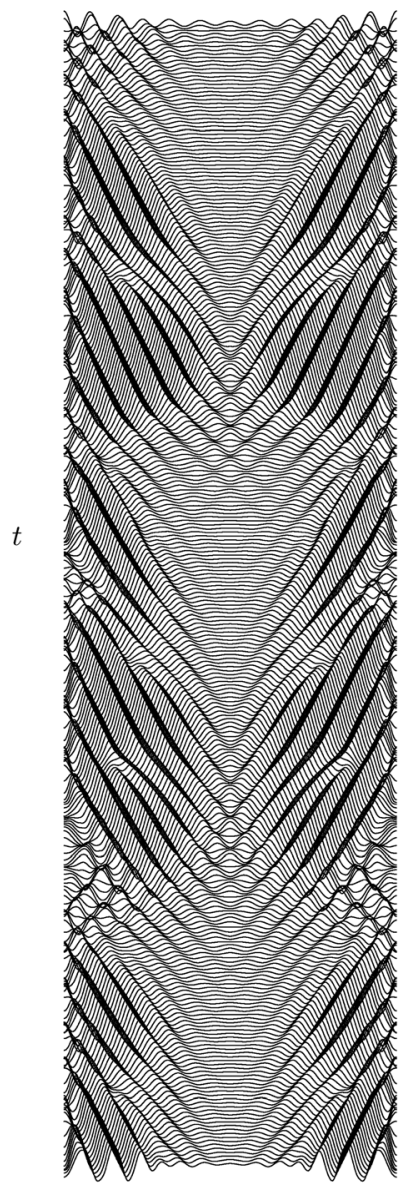

$x$

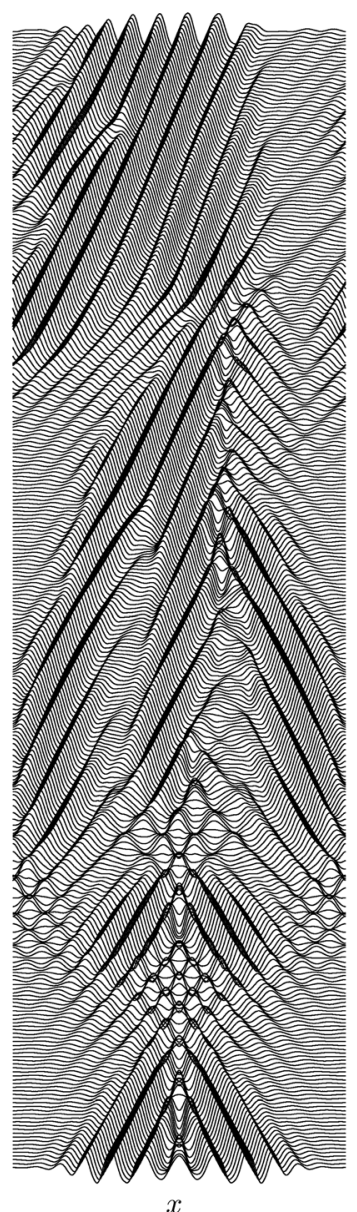

$x$

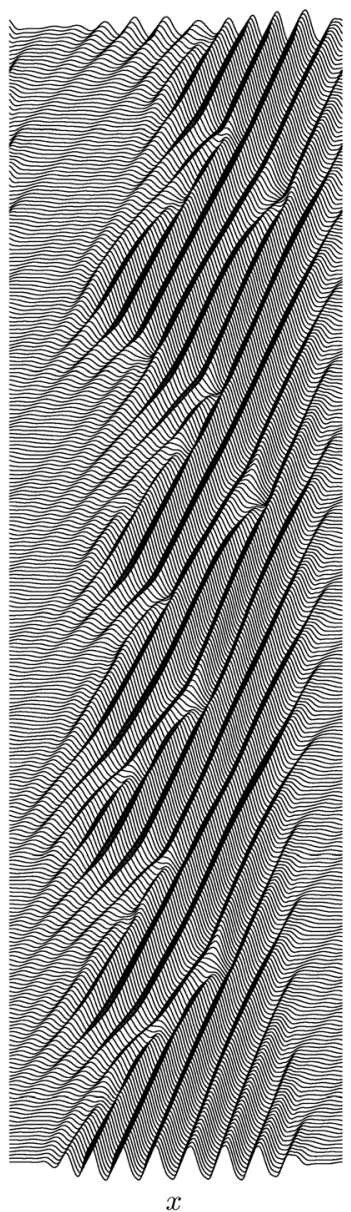

FIG. 14. Space-time plot with time increasing upward of the midplane vertical velocity $w(x, \quad z=1 / 2, \quad t)$ at $R a=2550$ in the time intervals $5 \leq t \leq$ $35,45 \leq t \leq 75$, and $85 \leq t \leq 115$, respectively, starting from a perturbation of the trivial state resembling the localized state labeled 5 in Figures 4 and 5. The solution first approaches an unstable symmetric orbit (left panel) with a source of left and right TW in the center $x=0$ and a sink at $x=\Gamma / 2$. The source turns into a sink and vice versa with increasing time (middle panel), followed by a symmetry-breaking transition leading to a stable but chaotic wave packet (right panel) consisting of right-traveling waves that drifts slowly to the right.
In contrast, time evolution of amplitude-stable convectons within the snaking region always leads to instability. The instability originates in the wings of the convectons and takes the form of growing traveling wave disturbances. The spatial inhomogeneity in the wings selects the propagation direction of the disturbance, resulting in waves that propagate towards the structure, growing as they do so. Since no fully developed TW are present at the Rayleigh numbers corresponding to the pinning region, the TW slow down as their amplitude approaches the amplitude of steady convection, and the system gradually settles into spatially periodic steady convection. Figure 16(a) shows the evolution at $R a=2732.0857$ of an unstable convecton located between solutions 2 and 3 on the $L_{10}^{1+}$ branch (see Figure 5), triggered by numerical perturbation only. Figure 16(b) shows the corresponding time series of the kinetic energy $E$ during the same time period. Although the kinetic energy increases it does not do so monotonically. The behavior shown is insensitive to the parity and width of the initial state. Similar evolution is observed to the right of the snaking region, i.e., for $R a>R a_{\text {right }}$, as well. These results suggest that for the parameter values employed, the conduction state is absolutely unstable. This hypothesis is confirmed by the stability analysis in Figure 17 which demonstrates, following Batiste et al., ${ }^{8}$ that the absolute instability threshold occurs at $R a=R a_{a b s} \approx 2540.2$. It follows that the conduction state is absolutely unstable with respect to traveling wave perturba- tions for $R a>2540.2$, a parameter range that includes the snaking region. Thus, all time-independent convectons located in the snaking region are expected to be unstable with respect to TW perturbations in their wings. As we have seen this does not mean that localized structures are absent, only that they cannot be time-independent. We remark that the convectons identified in binary mixture convection ${ }^{8}$ are located below the onset of absolute instability and, hence, in a parameter regime, where the instability of the conduction state is only convective. In this regime, any TW disturbance in the wings propagates away faster than it can grow and is absorbed by the convectons. ${ }^{8}$ Our study of the parameter dependence of $R a=R a_{a b s}$ reveals that $R a=R a_{a b s}$ decreases with decreasing $\tau$ but increases with increasing $R a_{S}$. Since the width of the snaking region increases with increasing $R a_{S}$ (i.e., with increasing subcriticality of periodic convection ${ }^{39}$ ), the possibility remains that for appropriate parameter values the snaking region may extend across $R a=R a_{a b s}$, thereby dramatically changing the stability properties of the convectons in this system.

\section{DISCUSSION}

In this paper, we have identified various types of spatially localized structures in doubly diffusive convection. In particular, we have computed both one-pulse and two-pulse states and showed that these are organized in parameter 


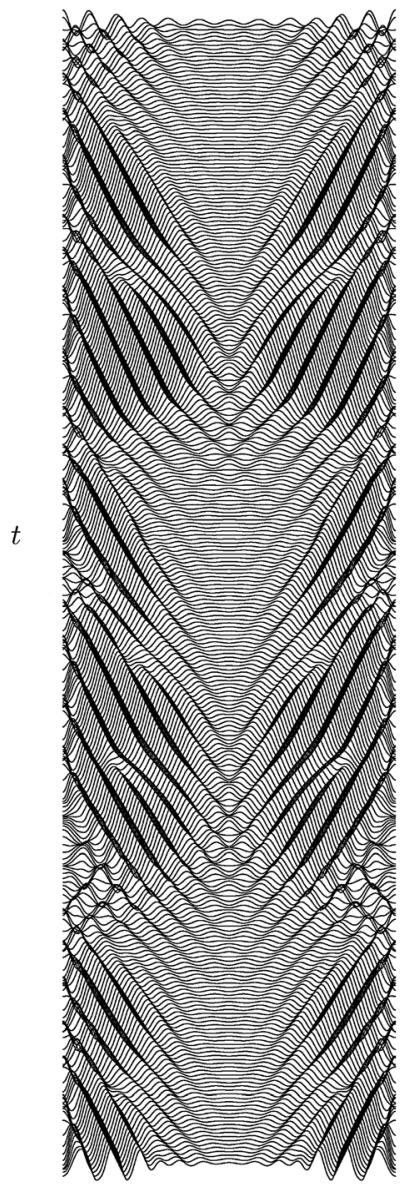

$x$

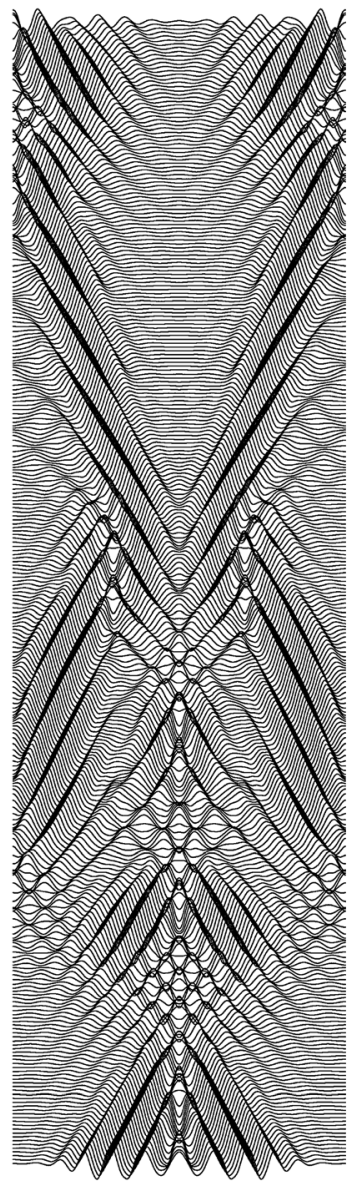

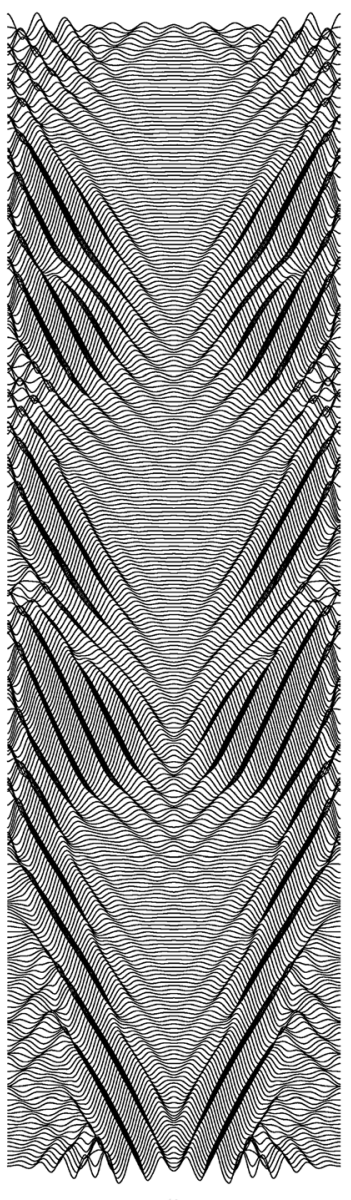

FIG. 15. Same as Figure 14 but with reflection symmetry imposed at $x=0$. space within a snaking or pinning region, precisely as occurs in other fluid systems such as binary fluid convection, ${ }^{8,12}$ natural doubly diffusive convection, ${ }^{29}$ and plane Couette flow. ${ }^{41}$ In contrast to binary fluid convection where concentration pumping in the horizontal by odd parity convectons modifies the width of the snaking region relative to that for the even parity convectons, both convecton types occupy the same pinning region and, hence, behave more like model systems such as the 35 Swift-Hohenberg equation. ${ }^{37} \mathrm{We}$ have related this observation to the absence of horizontal concentration pumping in the problem studied, a consequence of the different concentration boundary conditions imposed along the horizontal boundaries. We have also shown that for the parameter values used these states are unstable and attributed this observation to the fact that the pinning region lies in a parameter regime in which the conduction state is absolutely unstable. We have not, however, been able to perform our calculations for Lewis numbers of order $10^{-2}$, a value that is more representative of systems driven by parallel gradients of temperature and concentration, for which the threshold for absolute instability shifts to higher Rayleigh numbers. We have, in addition, computed several time-dependent but localized states, for Rayleigh numbers below the pinning region. Closer to the onset of the convective instability at $R a_{H}$, these states are dominated by advection at the group velocity. However, for $R a>R a_{a b s}$, the structures are dominated by the growth rate of the instability and, hence, by nonlinear effects. The structures identified differ from the dispersive chaos ${ }^{19}$ present close to $R a_{H}$ and represent spatially localized but chaotic traveling waves. Similar waves have been seen in direct numerical simulations in a closed large-aspect-ratio two-dimensional container at larger supercriticality ${ }^{42}$ and resemble the traveling wave packets observed in experiments. ${ }^{43}$ In periodic domains, these nonlinear wave packets drift in the direction of the phase velocity ( $s_{0}>0$, as confirmed below) but much more slowly. ${ }^{44}$ We mention that different types of localized waves, nonlinear compression waves traveling on a background of traveling waves, have also been identified in doubly diffusive convection. ${ }^{45}$

We have also found structures of source and sink type. Sources are localized structures that emit waves, while sinks arise at locations, where waves traveling in opposite directions collide. Such structures have been extensively studied in binary fluid convection. ${ }^{46}$ To the extent that they are representative of the behavior of doubly diffusive convection beyond the onset of convective instability our results suggest that sinks are more robust (see, e.g., Figure 13) than sources (see, e.g., Figure 14). These conclusions agree qualitatively with predictions using a pair of (supercritical) coupled complex Ginzburg-Landau (hereafter CCGL) equations, ${ }^{47}$ despite the fact that our traveling waves bifurcate weakly subcritically. We believe that this is because the TW branch turns around quickly towards larger Rayleigh numbers and, 


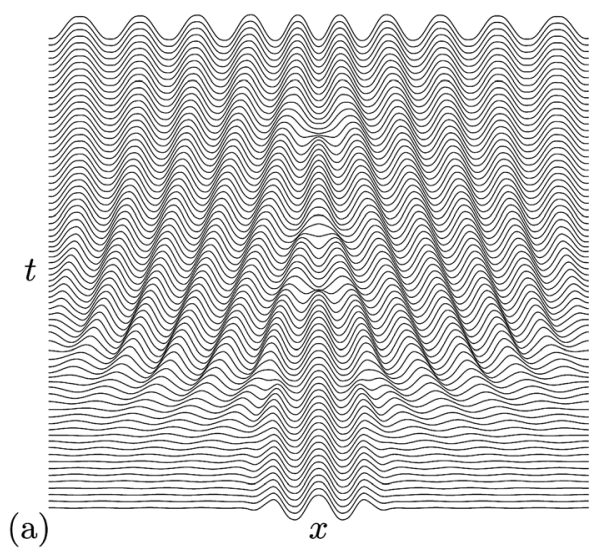

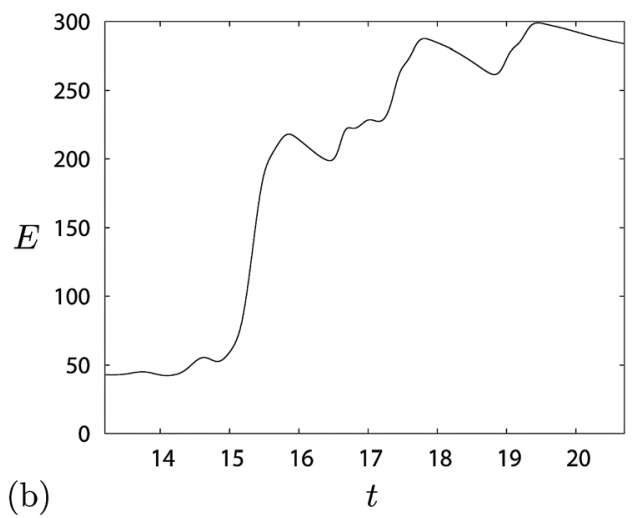

FIG. 16. (a) Space-time plot of the midplane vertical velocity $w(x, z=1 / 2, t)$ at $R a=2732.0857$ starting from a state between solutions 2 and 3 on the $L_{10}^{1+}$ convecton branch in Figure 5. (b) Evolution of the kinetic energy $E$ with time over the same time period as in (a). hence, comes to resemble the supercritical states considered in Ref. 47. The theory based on the CCGL equations reveals that as $\epsilon \equiv\left(R a-R a_{H}\right) / R a_{H}$ decreases steady sources lose stability and become time-dependent and potentially chaotic. A telling example illustrating the fragility of a source is provided in Fig. 23 of Ref. 46. In order to compare this prediction with our numerical simulations, we have evaluated the predicted critical value of $\epsilon$ for this instability, ${ }^{47,48}$

$$
\epsilon^{*}=\frac{s_{0}^{2}}{4\left(1+c_{1}^{2}\right)},
$$

using the group speed $s_{0}$ and the dispersion coefficient $c_{1}$ computed from the solution of the full linear stability problem by performing a suitable Taylor series expansion of both the critical Rayleigh number and the associated frequency about the critical wavenumber $k_{H}$ and critical frequency $\omega_{H}$ at the onset of the Hopf bifurcation. Scaled appropriately, we find that for our parameter values $s_{0} \approx 0.7862>0$, while $c_{1} \approx 0$ to the same accuracy, a result that is consistent with Figure 14 (right panel). Thus $\epsilon^{*} \approx 0.1545$, implying that (symmetric) sources should be unstable for $R a<R a^{*} \approx$ 2586. This prediction is consistent with our simulations which find an unstable source at $R a=2550$ (Figure 14).

In contrast, the sinks form by collision and if the fully developed TW are not modulationally unstable the sink may be a stable structure that must persist all the way down to $R a=R a_{H}$. Such sinks will be stationary if the incoming waves are related by reflection or will drift due to wavenumber and/or frequency mismatch between the incoming wavetrains. Of course, the CCGL description eliminates the basic wavetrains from the description, but it is clear that if these are reinstated and their interaction fully described there will be two types of sources and sinks between identical wavetrains traveling in opposite directions: a symmetric source, in which the waves are emitted simultaneously in both

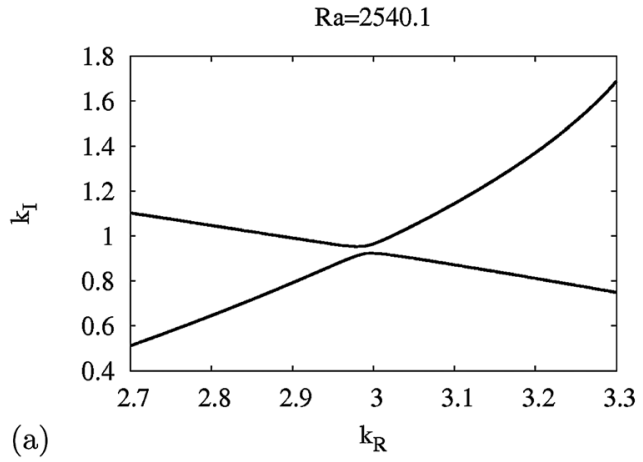

(a)

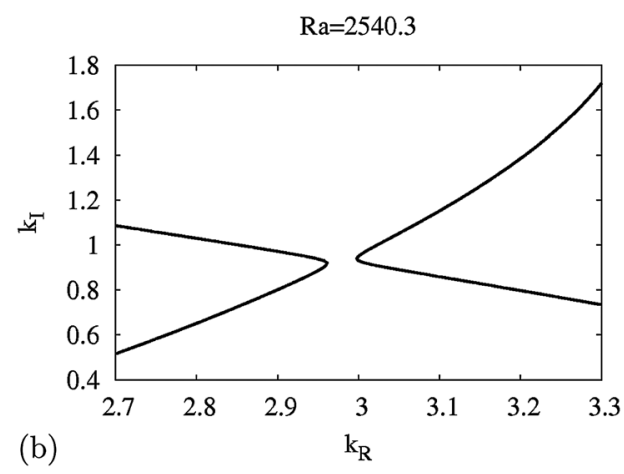

$\mathrm{Ra}=2540.1$

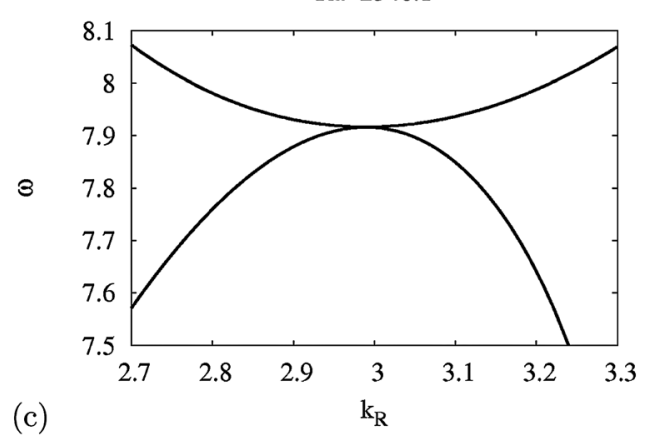

(b)

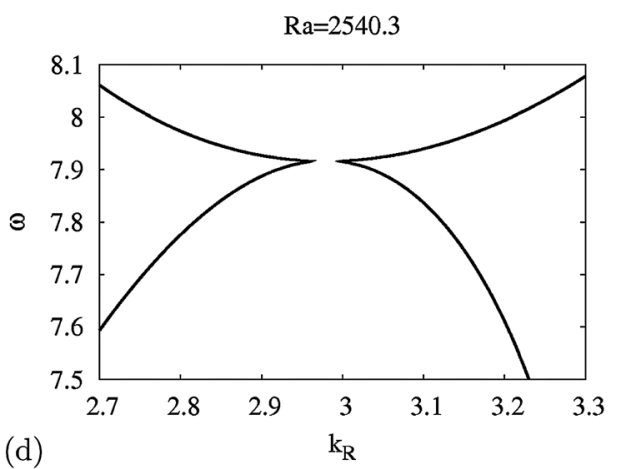

FIG. 17. The solution of the linear stability problem of the conduction state in complex wavenumber space $k \equiv k_{R}+i k_{I}$. The pinching that takes place between panels (a) and (b) identifies $R a=R a_{a b s} \approx$ 2540.2. Panels (c) and (d) show the corresponding wave frequency $\omega$. 
directions (i.e., in phase) and an antisymmetric source in which the waves are emitted exactly out of phase, with a wave emitted in one direction in the first half-period and in the opposite direction in the second half-period. States of the latter type resemble one-dimensional spiral waves. The same dichotomy arises for sinks. It is of interest, therefore, to remark that the source in Figure 14 is unsteady and symmetric, while the sink in Figure 13 is not only stable but also antisymmetric. The same is true of a similar sink computed a number of years ago, ${ }^{13,49}$ for different parameter values and stress-free lateral and as well as horizontal boundaries, indicating robustness of this type of sink (see also Ref. 50, Fig. 12). We mention that with stress-free boundary conditions, the bifurcation at $R a_{H}$ to TW is degenerate ${ }^{17-19}$ and the application of the CCGL description ${ }^{47}$ in the nonlinear regime is not rigorous. In view of the subcritical instability of the TW this is so in the present problem as well.

\section{ACKNOWLEDGMENTS}

This work was supported in part by the National Science Foundation under grant DMS-0908102 (E. K.) and by CNES under GdR MFA 2799 "Micropesanteur Fondamentale et Appliquée" (C. B. \& A. B.).

${ }^{1}$ J. S. Turner, "The coupled turbulent transports of salt and heat across a sharp density interface," Int. J. Heat Mass Transfer 8, 759 (1965).

${ }^{2}$ R. W. Schmitt, "Double diffusion in oceanography," Annu. Rev. Fluid Mech. 26, 255 (1994).

${ }^{3}$ W. R. Wilcox, "Transport phenomena in crystal growth from solution," Prog. Cryst. Growth Charact. Mater. 26, 153 (1993).

${ }^{4}$ U. Hansen and D. A. Yuen, "Nonlinear physics of double-diffusive convection in geological systems," Earth-Sci. Rev. 29, 385 (1990).

${ }^{5}$ E. Knobloch, "Nonlinear diffusive instabilities in differentially rotating stars," Geophys. Astrophys. Fluid Dyn. 22, 133 (1982).

${ }^{6}$ H. E. Huppert and D. R. Moore, "Nonlinear double-diffusive convection," J. Fluid Mech. 78, 821 (1976).

${ }^{7}$ D. R. Moore, J. Toomre, E. Knobloch, and N. O. Weiss, "Period doubling and chaos in partial differential equations for thermosolutal convection," Nature 303, 663 (1983).

${ }^{8}$ O. Batiste, E. Knobloch, A. Alonso, and I. Mercader, "Spatially localized binary-fluid convection,” J. Fluid Mech. 560, 149 (2006).

${ }^{9}$ A. Bergeon and E. Knobloch, "Periodic and localized states in natural doubly diffusive convection," Physica D 237, 1139 (2008).

${ }^{10}$ G. W. Hunt, M. A. Peletier, A. R. Champneys, P. D. Woods, M. A. Wadee, C. J. Budd, and G. J. Lord, "Cellular buckling in long structures," Nonlinear Dyn. 21, 3 (2000).

${ }^{11}$ J. Burke and E. Knobloch, "Homoclinic snaking: structure and stability," Chaos 17, 037102 (2007).

${ }^{12}$ D. Lo Jacono, A. Bergeon, and E. Knobloch, "Spatially localized binary fluid convection in a porous medium," Phys. Fluids 22, 073601 (2010).

${ }^{13}$ A. E. Deane, E. Knobloch, and J. Toomre, "Traveling waves in largeaspect-ratio thermosolutal convection," Phys. Rev. A 37, 1817 (1988).

${ }^{14}$ A. A. Predtechensky, W. D. McCormick, J. B. Swift, A. G. Rossberg, and H. L. Swinney, "Traveling wave instability in sustained double-diffusive convection," Phys. Fluids 6, 3923 (1994).

${ }^{15}$ J. D. Crawford and E. Knobloch, "Symmetry and symmetry-breaking bifurcations in fluid mechanics," Annu. Rev. Fluid Mech. 23, 341 (1991).

${ }^{16} \mathrm{E}$. Knobloch, D. R. Moore, J. Toomre, and N. O. Weiss, "Transitions to chaos in two-dimensional double-diffusive convection," J. Fluid Mech. 166, 409 (1986).

${ }^{17}$ E. Knobloch, A. E. Deane, J. Toomre, and D. Moore, "Doubly diffusive waves," Contemp. Math. 56, 203 (1986).

${ }^{18}$ A. E. Deane, E. Knobloch, and J. Toomre, "Traveling waves and chaos in thermosolutal convection," Phys. Rev. A 36, 2862 (1987).

${ }^{19}$ C. S. Bretherton and E. A. Spiegel, "Intermittency through modulational instability," Phys. Lett. A 96, 152 (1983).
${ }^{20}$ L. S. Tuckerman, "Steady-state solving via Stokes preconditioning: Recursion relations for elliptic operators," in 11th International Conference on Numerical Methods in Fluid Dynamics, edited by D. Dwoyer, M. Hussaini, and R. Voigt, Lecture Notes in Physics Vol. 323 (Springer, Berlin, 1989), p. 573.

${ }^{21}$ C. K. Mamun and L. S. Tuckerman, "Asymmetry and Hopf bifurcation in spherical Couette flow," Phys. Fluids 7, 80 (1995).

${ }^{22}$ G. E. Karniadakis, M. Israeli, and S. A. Orszag, "High-order splitting methods for the incompressible Navier-Stokes equations," J. Comp. Phys. 97, 414 (1991).

${ }^{23}$ A. Bergeon and E. Knobloch, "Natural doubly diffusive convection in three-dimensional enclosures," Phys. Fluids 14, 92 (2002).

${ }^{24}$ D. Funaro, Polynomial Approximation of Differential Equations (Springer, New York, 1991).

${ }^{25}$ M. Deville, P. F. Fischer, and E. Mund, High-order Methods for Incompressible Fluid Flow (Cambridge University Press, New York, 2002).

${ }^{26}$ R. Peyret, Spectral Methods for Incompressible Viscous Flow, Applied Mathematical Sciences Vol. 148 (Springer, New York, 2002).

${ }^{27}$ A. Quarteroni and A. Valli, Domain Decomposition Methods for Partial Differential Equations (Oxford Science Publications, 1999).

${ }^{28}$ C. Canuto, M. Y. Hussaini, A. Quarteroni, and T. A. Zang, Spectral Methods in Fluid Dynamics (Springer, New York, 1988).

${ }^{29}$ A. Bergeon and E. Knobloch, "Spatially localized states in natural doubly diffusive convection," Phys. Fluids 20, 034102 (2008).

${ }^{30}$ A. Bergeon, J. Burke, E. Knobloch, and I. Mercader, "Eckhaus instability and homoclinic snaking," Phys. Rev. E 78, 046201 (2008).

${ }^{31}$ P. D. Woods and A. R. Champneys, "Heteroclinic tangles and homoclinic snaking in the unfolding of a degenerate Hamiltonian-Hopf bifurcation," Physica D 129, 147 (1999).

${ }^{32} \mathrm{P}$. Coullet, C. Riera, and C. Tresser, "Stable static localized structures in one dimension," Phys. Rev. Lett. 84, 3069 (2000).

${ }^{33}$ M. Beck, J. Knobloch, D. J. B. Lloyd, B. Sandstede, and T. Wagenknecht, "Snakes, ladders and isolas of localized patterns," SIAM J. Math. Anal. 41, 936 (2009).

${ }^{34}$ E. Knobloch, "Convection in binary fluids," Phys. Fluids 23, 1918 (1980).

${ }^{35}$ P. Kolodner, C. M. Surko, and H. Williams, "Dynamics of traveling waves near the onset of convection in binary fluid mixtures," Physica D 37, 319 (1989).

${ }^{36}$ V. Steinberg, J. Fineberg, E. Moses, and I. Rehberg, "Pattern selection and transition to turbulence in propagating waves," Physica D 37, 359 (1989).

${ }^{37}$ J. Burke and E. Knobloch, "Snakes and ladders: localized states in the Swift-Hohenberg equation," Phys. Lett. A 360, 681 (2007).

${ }^{38}$ J. Burke and E. Knobloch, "Multipulse states in the Swift-Hohenberg equation," Discrete Contin. Dyn. Syst. (Suppl.), 109 (2009).

${ }^{39}$ A. Alonso, O. Batiste, E. Knobloch, and I. Mercader, "Convectons," in Localized States in Physics: Solitons and Patterns, edited by O. Descalzi, M. Clerc, S. Residori, and G. Assanto (Springer, Berlin, 2010), p. 109.

${ }^{40}$ C. Martel, E. Knobloch, and J. M. Vega, "Dynamics of counterpropagating waves in parametrically forced systems," Physica D 137, 94 (2000).

${ }^{41}$ T. M. Schneider, J. F. Gibson, and J. Burke, "Snakes and ladders: Localized solutions of plane Couette flow," Phys. Rev. Lett. 104, 104501 (2010).

${ }^{42} \mathrm{O}$. Batiste and E. Knobloch, "Simulations of oscillatory convection in ${ }^{3} \mathrm{He}-{ }^{4} \mathrm{He}$ mixtures in moderate aspect ratio containers," Phys. Fluids 17, 064102 (2005).

${ }^{43}$ P. Kolodner, "Stable and unstable pulses of traveling-wave convection," Phys. Rev. A 43, 2827 (1991).

${ }^{44}$ W. Barten, M. Lücke, M. Kamps, and R. Schmitz, "Convection in binary fluid mixtures. II. Localized traveling waves," Phys. Rev. E 51, 5662 (1995).

${ }^{45}$ A. Spina, J. Toomre, and E. Knobloch, "Confined states in large-aspectratio thermosolutal convection,” Phys. Rev. E 57, 524 (1998).

${ }^{46} \mathrm{P}$. Kolodner, "Extended states of nonlinear traveling-wave convection. II. Fronts and spatiotemporal defects," Phys. Rev. A 46, 6452 (1992).

${ }^{47}$ M. van Hecke, C. Storm, and W. van Saarloos, "Sources, sinks and wavenumber selection in coupled CGL equations and experimental implications for counter-propagating wave systems," Physica D 134, 1 (1999).

${ }^{48}$ P. Coullet, T. Frisch, and F. Plaza, "Sources and sinks of wave patterns," Physica D 62, 75 (1993).

${ }^{49}$ A. E. Deane, E. Knobloch, and J. Toomre, "Erratum: Traveling waves in large-aspect-ratio thermosolutal convection [Phys. Rev. A 37, 1817 (1988)]," Phys. Rev. A 38, 1661 (1988).

${ }^{50}$ L. Pastur, M.-T. Westra, and W. van der Water, "Sources and sinks in 1d traveling waves," Physica D 174, 71 (2003). 